\title{
El aprovechamiento agrícola de las zonas húmedas: la introducción del arcaduz en el sureste de Al-Andalus (siglos VIII y IX)'
}

\author{
Sonia Gutiérrez Lloret*
}

"Pero aún no ha llegado esa hora en que las aguas subirán hacia arriba"

J. L. Sampedro (Octubre, Octubre)

Arcaduz es una de las voces que designan en lengua castellana el vaso de metal o más comúnmente de cerámica, que sirve para sacar agua de pozos y ríos, atado con otros a una maroma doble que descansa sobre la rueda de la noria o cenia. ${ }^{2}$ La utilización generalizada de este término entre los arqueólogos medievalistas, en detrimento de otros de uso más frecuente como el de cangilón, se debe a la formalización semántica propuesta en su día por Guillermo Roselló, quien optó por dicha denominación en razón de su origen árabe -qādūs- y su mención expresa en fuentes andalusíes del siglo $\mathrm{XI} .^{3}$

Sin embargo, a pesar de que este recipiente cerámico aparecía con abundancia en los yacimientos medievales no siempre resultaba posible establecer su cronología, considerándose a menudo un tipo funcional caracterizado por su escasa variabilidad morfológica a lo largo de los siglos (BAZZANA et alii, 1987, 78). El primer intento riguroso de superar esta imprecisión cronológica procede de un trabajo de A. Bazzana, S. Climent e Y. Montmessin sobre el yacimiento valenciano de "Les Jovades", en Oliva, y más concretamente del estudio arqueológico de un pozo de noria del que proceden más de mil vasos, de los que un centenar están completos o resultan reconstruibles en su forma. ${ }^{4}$ Las piezas de Oliva -agrupadas en ocho formas designadas por letras en orden alfabético correlativo- se caracterizaban por presentar dos puntos de fijación a la cadena, uno inmediatamente debajo del borde y otro en la mitad del cuerpo, mientras que la morfología general de la base variaba de unos tipos a otros. Esta clasificación morfológica fue acompañada de un intento de ofrecer las primeras secuencias cronológicas, al proponerse la mayor antigüedad de las formas con base anular -formas $A$ y B- $y$ de las fondo puntiagudo -formas $F$ y G(siglos $X-X I)$, frente a las cilíndricas de base plana y perforada, ya características de los siglos XIII, XIV y XV.

De hecho, tanto la forma de base plana como la puntiaguda, sobre todo ésta última, eran conocidas y estaban documentadas en

* Universidad de Alicante. Depto. de Prehistoria y Arqueología

I Este trabajo se presentó al coloquio Historia y Medio Físico (Agricultura y regadío en Al-Andalus, Síntesis y problemas), celebrado en Almería los días 9 y 10 de Junio de 1995. El texto original se reproduce íntegramente. Se añaden tres figuras (I a 3) y la lámina I, con sus correspondientes llamadas en el texto y la pertinente actualización bibliográfica de los trabajos citados en prensa.

2 s. v. cangilón, Diccionario de la Lengua Española (20ª ed., 1984).

3 G. ROSELLÓ, 1978, y más recientemente 1991, pp. 21 y 149. Véase también BAZZANA, 1979, p. 163.

4 BAZZANA et alii, 1987, 67 y ss. Además de esta obra, el mismo año se publicó a modo de avance un artículo firmado por A. BAZZANA: "Una noria árabe en la huerta de Oliva (Valencia)", I I C.A.M.E., II, 42I-32. 
diversos yacimientos de al-Andalus, ${ }^{5}$ si bien carentes casi siempre de un contexto estratigráfico datable con relativa precisión. Este panorama comenzó a cambiar a finales de la década de los ochenta, cuando las excavaciones sistemáticas en el yacimiento almeriense de Pechina exhumaron los primeros arcaduces fechables con fiabilidad en el siglo IX. Los arcaduces baŷȳāníes emirales son de tendencia cilíndrica, base plana y dos escotaduras muy marcadas en el cuerpo (ACIÉN y MARTínEZ, 1989, 134). En un trabajo de síntesis posterior sobre los tipos cerámicos del sureste de al-Andalus, incluyendo en este caso únicamente las actuales provincias de Almería, Málaga y Granada, ${ }^{6}$ los autores señalan la evolución hacia formas con bases aguzadas ya en el califato, llegando en época nazarí a las bases semiesféricas. Curiosamente, la seriación propuesta en el caso de Andalucía oriental difiere totalmente de la obtenida a partir de la noria de Oliva, en la que las bases puntiagudas se consideraban más antiguas que los fondos planos, si bien en este caso presentaban siempre la perforación basal que está totalmente ausente en las piezas emirales de Pechina.

Aunque la noria de Oliva no proporcionó una estratificación completamente fiable (BAZZANA et alii, 1987, 104), no existe ninguna razón irrefutable para dudar de la validez general de la evolución tipológica propuesta, si bien matizando la posible perduración de las formas de base puntiaguda con posterioridad al siglo XI, puesto que se documentan en el contexto de abandono del baño de la calle Polo de Medina en Murcia, fechado entre el segundo y el tercer cuarto del siglo XIII (POZO, 1991, figs. 37 y 38). Por esta razón, las discrepacias observadas en las seriaciones de arcaduces obtenidas en estos dos yacimientos de la fachada mediterránea de al-Andalus parecen ya indicar por si mismas las, por otro lado lógicas, diferencias regionales en las evoluciones tipológicas del mobiliario cerámico y contribuyen a desterrar definitivamente el tópico funcionalista de su inmutabilidad morfológica y la subsiguiente imposibilidad de datación.

De forma paralela, en 1988 dábamos a conocer una nueva forma de arcaduz cerámico definida a partir de los numerosos ejemplares hallados en un yacimiento alicantino próximo a la desembocadura del río Segura, el Cabezo del Molino en Rojales (GUTIÉRREZ LLORET, 1988, 210-I) (fig. I, 6). Las piezas que entonces presentamos procedían de un expolio clandestino que afortunadamente pudo ser abortado, recuperándose los materiales exhumados que quedaron depositados en el museo local. El hallazgo presentaba una gran homogeneidad en pastas y tipos de cerámica común, entre la que las producciones vidriadas brillaban por su ausencia; estaba formado por marmitas de base plana modeladas a mano (fig. 2, 7 y 8 ), ollas (fig. 2, 9), candiles de piquera corta (fig. 2 , I0 y | I), jarritos y jarras torneados, destacando los decorados con filetes de óxido de hierro (Fig. 3, 2 y 3), junto con un importante conjunto de vasos que interpretamos como arcaduces (fig. 3, I). Este último tipo resultó el de más difícil atribución puesto que a pesar de disponer de casi medio centenar de bases, ninguna pieza permitía la reconstrucción de un perfil completo (lám. I).

No obstante, aún desconociendo la forma del borde, la parte conservada de la pieza ya apuntaba lo que por entonces parecía un rasgo disonante y que en más de una ocasión motivó que algunos investigadores cuestionaran dicha atribución: parecía evidente que el punto de fijación inferior se situaba en el fondo del recipiente, aprovechando el resalte de la base, y no en la parte media del cuerpo, puesto que carecían del característico estrangulamiento. A

\footnotetext{
5 Sin ánimo de ser exhaustivos podemos citar los ejemplos de Almadén y la Alcazaba de Málaga (BOSCH y CHINCHILLA, I987, 497), el alfar de Bezmiliana en Málaga (ACIÉN, 1990), Sevilla (CARRASCO, 1987, 53I y ss.), Burriana, Denia, Torre Bufilla, la ciudad de Valencia (apud BAZZANA et alli, 1987, 100-104), Son Mosson en Mallorca (ROSSELLO, 1978, 31।-2) o Madrid (RETUERCE, 1990, 160). Es muy frecuente que los ápices de ciertos arcaduces presenten un acabado característico, consistente en un facetado producido al cortar el barro con la intención aparente de aguzar aún más el extremo puntiagudo del vaso; dicha técnica se aprecia en diversos ejemplares de Bezmiliana, Mallorca y Sevilla, siendo este rasgo, junto con el marcado torneado de sus paredes, uno de los que permiten reconocer los fragmentos amorfos de estos recipientes.

6 ACIÉN et alii; comunicación inédita presentada al V Colloque International sur la céramique médiévale en Méditerranée Occidentale (Rabat, |99|), gentileza de los autores.
} 
pesar de lo novedoso de este dato en comparación con las series andalusíes conocidas, la existencia de paralelos etnograficos egipcios, publicados por T. Schiøler (1973, 97 y ss.), apoyaba nuestra hipótesis (GUTIÉRREZ LLORET, 1988, 210). De otro lado, el aspecto más importante que se derivaba de esta atribución era la temprana cronología del lote de materiales, para el que proponíamos una datación provisional entre la segunda mitad del siglo VIII y la primera del IX (GUTIÉRREZ LLORET, 1988, 245), lo que en caso de confirmarse convertiría a los arcaduces del Bajo Segura en los más antiguos conocidos.

Lo que en 1988 se planteó como una tímida e inicial hipótesis de identificación de materiales emirales, se verificó en el marco de un estudio exhaustivo de la cultura material y del poblamiento emiral y califal de las tierras de Tudmîr. De un lado, se pudo comprobar la frecuente aparición de arcaduces de similares características en numerosos yacimientos rurales del Bajo Segura e incluso en algunos urbanos como la ciudad de Murcia y de otro, fue posible reconstruir los primeros perfiles completos, sugiriendo una evolución tipológica entre las formas emirales y las califales. ${ }^{8} \mathrm{De}$ esta forma, estamos en condiciones de proponer un tipo de arcaduz ${ }^{9}$ de cronología emiral (mediados del siglo VIII y siglo IX), documentado en diversos asentamientos del valle fluvial del río Segura en su tramo inferior. Se trata de un recipiente de tendencia troncocónica con una amplia base irregular, plana o ligeramente convexa, una marcada inflexión en el hombro y borde exvasado, ligeramente engrosado. ${ }^{10}$ Las pastas son bastas, de color claro y tonalidad variable, de textura medianamente compacta y blanda, con abundante desgrasante mineral de gran tamaño. Las piezas presentan en general una factura tosca $y$ descuidada, con huellas del hilo de corte en la base y las estrías de torno muy marcadas, lo que les confiere un característico aspecto moldurado. Tanto la base como la parte baja del cuerpo presentan a menudo señales de cortes destinados seguramente a aligerar peso y realzar el entalle de la base, que hace las veces de anclaje inferior. El punto de fijación superior se sitúa en la parte alta del vaso, justo en el estrangulamiento del hombro, mientras que el inferior, y ésta es la principal novedad respecto a otros ejemplares andalusíes de alta cronología como los de Pechina, aprovecha el realce de la base, careciendo por tanto de las características escotaduras en la parte media del cuerpo (fig. 4.2).

Al igual que ocurre con los ejemplares emirales de Pechina, las bases de los arcaduces del Bajo Segura nunca aparecen perforadas. Como señala el agronomo andalusí del siglo XII Ibn al-CAwām, citando a Abu al-Jayr, dicha perforación favorece la salida del aire en el momento en el que los arcaduces se introducen en el agua, impidiendo que se ladeen y golpeen entre sí, y permite que los vasos llenos que no han alcanzado el final del recorrido se vacien cuando la noria se detiene, evitando el exceso de peso y el retroceso consiguiente de la rueda." La ausencia de esta característica en los ejemplares más antiguos, tanto emirales como califales, parece demostrar que la perforación practicada en la base de los cangilones debió ser una solución tecnológica a los problemas de rendimiento de las norias, introducida en fechas más recientes (quizá a finales del siglo XI o ya en el XII).

\footnotetext{
7 Este trabajo, que constituyó mi tesis de doctorado defendida en Alicante en 1992, se halla ahora en curso de publicación con el título La Cora de Tudmīr: de la antigüedad tardía al mundo islámico, Casa de Veláquez-Instituto "Juan Gil-Albert".

8 Cfr. GUTIÉRREZ LLORET, 1993, pp. 44-59, fig. 7.16 para la forma emiral y 10.1-2 para las versiones califales

9 Forma T32.I. La argumentación arqueológica que confirma la temprana datación emiral del tipo en función de los contextos materiales a los que se asocia, excede el marco de esta comunicación, por lo que remito a la seriación cronotipológica contenida en la obra citada en la nota 7.

10 El diámetro de las bases oscila entre 7 y $10 \mathrm{~cm}$., mientras que el de la boca se encuentra entre 22 y 24 cm. Según las reconstrucciones, la altura aproximada se halla entre los 25 y los $30 \mathrm{~cm}$. La capacidad de los ejemplares del Cabezo del Molino resulta imposible de calcular por su estado fragmentario pero la de algunos ejemplares murcianos del siglo $X$ se ha establecido en torno a los 1800-2000 c. c. (MUÑOZ LÓPEZ, 1992, 182)

I L Libro de agricultura de IBN al- ${ }^{C}$ AWĀM, edición y traducción de J. A. Banquieri, Madrid I802 (ed. facs. I988), p. I47.
} 
A tenor de las pastas y las características formales de las piezas en cuestión se trata seguramente de producciones locales, realizadas en uno o varios talleres del Bajo Segura. De hecho, recientemente ha sido dado a conocer un interesante horno cerámico de la ciudad de Murcia, situado en la calle Cortés, en el barrio de San Nicolás, un área extramuros del supuesto recinto emiral-califal de Madina Mursiya, caracterizado por la abundancia de áreas alfareras. Entre los restos de la producción abandonada en el interior de la cámara de cocción del horno se hallaron cuatro jarritas y trece arcaduces de similares características a los aquí estudiados, si bien algo más modernos ya que se fechan entre la segunda mitad del siglo $X y$, a lo sumo, las primeras décadas del XI (MUÑOZ LÓPEZ, 1992, 180-2, lám. 5, figs. 3.5 y 3.6). Estas producciones murcianas son, como luego veremos, las herederas de las que aquí presentamos, por lo que es necesario suponer que los alfares urbanos de Murcia incorporan a sus repertorios, a lo largo del siglo $X$, formas de gran tradición en el ambiente rural del Bajo Segura, desplazando paulatinamente a los centros locales de producción, que las venían fabricando ya en contextos cronológicos anteriores a la fundación de la propia madinna.

De hecho, la concentración de arcaduces en el Cabezo del Molino sugiere la posibilidad de que se trate de un centro de producción local. El yacimiento arqueológico ocupa parte de la cima y laderas de un cerro próximo al río, por lo que no puede explicarse la gran concentración de arcaduces, realmente espectacular si se compara con la escasa potencia de la estratificación, por la inmediatez de un pozo de noria, que lógicamente se situaría en el llano aluvial. De otro lado, la excavación de urgencia realizada en 1990 en las inmediaciones de la zona expoliada ${ }^{12}$ puso en evidencia la inexistencia de estructuras de ocupación, excepción hecha de una tumba de inhumación de rito preislámico y de una cubeta tallada en la roca con la impronta de un pilar central, destinada posiblemente a un uso industrial indeterminado, si bien se detectaron numerosas bolsadas de material constructivo (ladrillos decorados con digitaciones, fragmentos de opera signina y tegulae, junto con dos fragmentos de toscas columnas de piedra arenisca) y cerámica (principalmente un homogéneo lote de cerámica emiral y algunos materiales tardorromanos. ${ }^{13}$ Con los datos actuales parece posible que los restos detectados correspondan a un área de vertidos procedente de un asentamiento rural del siglo VIII, de origen anterior (a juzgar por la aparición de materiales romanos y las características del enterramiento), y posiblemente productor de su propia cerámica.

Los arcaduces del Bajo Segura responden por tanto a una forma emiral que perdura en el califato transformándose morfológicamente. De hecho, esta evolución formal sugerida en el encuentro de Salobreña de 1990 a partir de materiales procedentes de la excavación del alfar antiguo de San Nicolás, pero no producidos en él (GUTIÉRREZ LLORET, 1993, fig. 10.1), halla su confirmación en el horno recientemente publicado de la calle Cortés de Murcia. En mi opinión, a lo largo del siglo $X$ el tipo de arcaduz emiral caracterizado por la fijación inferior en el reborde de la base plana o convexa de gran diámetro -entre 10 y $8 \mathrm{~cm}$.- (fig. 4.2), tiende a una paulatina reducción de la base, que alcanza en los ejemplares murcianos de mediados del siglo $X$ los $5 \circ 6 \mathrm{~cm}$ (fig. 4.3 y 4), hasta configurar un pivote o botón macizo patente en algunos ejemplares de la Rábita de Guardamar (fig. 4.6), fechables en contextos califales avanzados (GUTIÉRREZ LLORET, 1993, fig. 10.2). El paso siguiente será, como ha señalado acertadamente F. Muñoz López (1992, 182), el desplazamiento del anclaje inferior hacia la parte central del arcaduz, consolidado ya en el siglo XI y generalizado a partir de entonces, que supone una clara ruptura morfológica con la tradición formal de estos primeros ejemplares y deviene en su desaparición definitiva.

\footnotetext{
12 Dirigida por Pilar Beviá al objeto de delimitar la extensión del yacimiento arqueológico.

13 En concreto una moneda de la segunda mitad del siglo IV reutilizada como colgante, un fragmento de ánfora ibérica, cerámica africana de cocina (tapadera tipo "Ostia II"), un fragmento de cerámica narborense (tipo Rigoir 6; fig. 2,3) y algunos fragmentos de terra sigillata africana tardía (tipo D), entre los que destacan un borde del tipo Hayes 61 B (fig. 2,I) y algunos fragmentos de lucernas (fig. 2,4 a 6). El estudio definitivo de la excavación está siendo realizado por P. Beviá y yo misma.
} 
Ahora bien, la identificación de un arcaduz andalusí temprano, seguramente el más antiguo documentado arqueológicamente hasta el momento, tiene importantes implicaciones históricas entre las que las tipológicas no son precisamente las más significativas. Los arcaduces documentados en los asentamientos emirales del Bajo Segura, en tanto que artefactos tecnológicos, informan de la introducción a mediados del siglo VIII de las ruedas hidráulicas de tiro o "de sangre", es decir aquellas activadas por tracción animal, y de las prácticas de riego con ellas relacionadas (fig. 4.I); de otro lado, el desarrollo de dichas prácticas hidráulicas es consecuencia del diseño de una estrategia social de producción campesina, basada en la explotación de pequeños agrosistemas de regadío.

Aunque el principio de las máquinas elevadoras de agua era conocido en la antigüedad clásica y el uso de recipientes cerámicos en las ruedas hidráulicas se introdujo por primera vez en el Egipto romano de principios del siglo IV, ${ }^{14}$ la generalización de las norias o cenias de tiro con arcaduces, usadas para extraer agua de pozos, parece producirse en época islámica. De hecho, los arcaduces cerámicos no se documentan en los registros arqueológicos del sureste de la Península Ibérica con anterioridad al siglo VIII, ${ }^{15}$ razón por la que parece lógico deducir que la cenia pudo ser una innovación tecnológica introducida por las poblaciones árabes y beréberes y divulgada tras la conquista de al-Andalus. Curiosamente en el caso del Bajo Segura estos arcaduces se documentan, si bien no de forma tan espectacular como en el Cabezo del Molino, en la mayoría de los asentamientos emirales situados en las pequeñas elevaciones que perimetran las zonas pantanosas, características del tramo inferior del río. Tanto las escasas referencias documentales como la investigación arqueológica desarrollada en la zona, sobre cuyos pormenores sería prolijo extendernos aquí, ${ }^{16}$ sugieren el origen indígena de las poblaciones que ocupan dichos asentamientos, siendo la mecánica que explica el proceso de orden social. Se trata de sectores sociales desposeídos, procedentes de medios urbanos y rurales cada vez más feudalizantes, que ocupan áreas marginales pantanosas o agrestes donde resulta más fácil escapar al creciente control económico de los grupos privilegiados, desarrollando formas de economía campesina basadas en una estrategia productiva diversificada $y$, por tanto, equilibrada, que combina la agricultura con otras prácticas productivas complementarias o alternativas, como la caza, pesca o la recolección forestal. ${ }^{17}$

Sin embargo, desde fecha muy temprana -mediados del siglo VII- la aparición de arcaduces indica que las prácticas agrícolas de estas comunidades debieron enriquecerse con la introducción de nuevas técnicas de regadío, basadas en la elevación del agua de pozos mediante cenias con cadenas de cangilones. El desarrollo de estos pequeños agrosistemas de regadío de alto rendimiento se explica, en nuestra opinión, por la relación de las comunidades indígenas con los nuevos contingentes demográficos llegados a la Península tras su conquista y la relativamente rápida integración social patente en los numerosos matrimonios mixtos. En el caso del territorio que nos ocupa, dicha práctica se ejemplifica en el matrimonio de la hija de Teodomiro con un ŷundí de origen egipcio, que recibió en concepto de dote dos explotaciones rurales situadas en el Bajo Segura. ${ }^{18}$ De esta forma, las comunidades

14 Según T. Glick, $(1988,65)$ citando a OLESON, J.P. (1984): Greek and Roman Mechanical Water-Lifting Devices: The History of a Technology, Toronto, pág. 354. Véase también CARO BAROJA, 1983, 255 y ss.

15 Aun cuando el sistema de polea con cangilones fué ya descrito por Vitrubio (Libro X, IX, 23), las ruedas hidráulicas de drenaje atestiguadas en las explotaciones mineras hispanas fueron generalmente de compartimentos; de otro lado, los cangilones conocidos eran de cobre, como indica Vitrubio, con capacidad de un congio (3,50 litros) y no de cerámica (LUZÓN, I968, III).

16 Sobre el particular puede verse, entre otros, GUTIÉRREZ LLORET,1992, así como La cora de Tudmīr: de la antiguedad tardía al mundo islámico (e.p.).

17 GUTIÉRREZ LLORET, (1995): "El origen de la huerta de Orihuela entre los siglos VII y Xl: una propuesta arqueológica sobre la explotación de las zonas húmedas del Bajo Segura", Arbor, , 86 y ss.

18 Sobre las alquerías de Tarsa y Tall al-Jațāb, situadas a tres millas de Elche y ocho de Orihuela respectivamente, que según al'U ría con el asentamiento del Cabezo de la Fuentes en Albatera puede verse GUTIÉRREZ LLORET, e.p. y 1995 , pp. 85-6. 
indígenas debieron entrar en contacto con las nuevas poblaciones -en el caso de Tudmìr, ŷundíes egipcios- incorporando una nueva tecnología agraria, especialmente adecuada para la explotación de los bordes de los marjales con un alto nivel freático. Este proceso de uniformización tecnológica, que supone el diseño de los primeros espacios hidráulicos, corre parejo a un paulatino proceso de integración social. De esta forma, los arcaduces como artefactos cerámicos introducidos y totalmente ajenos a las tradiciones culturales preislámicas, adquieren una nueva dimensión, convirtiéndose en valiosos indicadores del proceso de aculturación de las poblaciones indígenas, en suma de su islamización social.

De otro lado y teniendo en cuenta que los únicos paralelos conocidos para el peculiar sistema de anclaje de los arcaduces del Bajo Segura proceden de Egipto ${ }^{19}$ (fig. 4.5 y lám. II), no deja de ser sugerente relacionar ambos fenómenos y recurrir al origen de los introductores -árabes orientales, egipcios o bereberes magrebíes- para explicar las diferentes opciones morfológicas que caracterizan a los arcaduces emirales. Sin embargo, por más tentadora que parezca la hipótesis no resulta menos "peligrosa", al incurrir en el argumento etnográfico que, no conviene olvidarlo, nunca es probatorio y siempre encierra el riesgo del anacronismo.

Por último, sólo resta incidir en un valor añadido, quizá el más importante de todos, que hace aún más significativo el estudio de estos arcaduces. Es probable que su reconocimiento sea el único método arqueológico que permita documentar la existencia de estos primitivos -en tanto primeros en el orden cronológico de formación, que no rudimentarios- sistemas hidraúlicos basados en el riego de las áreas perimetrales de los marjales, mediante ingenios de elevación del agua. La compleja caracterización física de los espacios regados en los llanos de inundación, sabiamente estudiada por los geógrafos $\mathrm{V}$. M. Rosselló Verger (1989) y J. Mateu (1989), puede condicionar enormemente e incluso llegar a impedir la descripción arqueológica minuciosa de los espacios agrarios del Bajo segura, recientemente reclamada por $\mathrm{H}$. Kirchner y C. Navarro, ${ }^{20}$ al menos en lo tocante a las áreas de cultivo de los asentamientos emirales. Esta dificultad no emana de una preferencia metodológica ni menos aún del denuesto de las técnicas del trabajo de campo derivadas de la arqueología extensiva, sino de la propia caracterización geomorfológica de los espacios en estudio.

Estos agrosistemas se desarrollaron en los bordes de los sectores deprimidos y anegados del valle fluvial del río Segura. Como J. Mateu señala, los llanos de inundación son superficies de activa acumulación aluvial, caracterizados por una importante acreción vertical y lateral (MATEU, 1989, 170 y ss.), favorecida además por la intensa deforestación antropogénica de los períodos históricos más recientes. Aunque estos procesos de aluvionamiento nunca son uniformes en toda la llanura, es innegable que pueden alcanzar espesores considerables en ciertos puntos. El problema no afecta tanto a las áreas de residencia, para las que conscientemente se seleccionan zonas no inundables, como a los campos de cultivo que ocupan las superficies donde la avenida permite la recarga del acuífero y la renovación de los nutrientes del suelo por la deposición de limos fértiles (ROSSELLÓ VERGER, 1989, 245). Ésta y no una preferencia arqueológica tendenciosa es la razón por la cual el asentamiento emiral del Bajo Segura ha sido reconocido a partir de las primeras, las áreas de residencia, que son más evidentes. Aunque la cuestión del aluvionamento debe ser ponderada por los expertos, en este caso los geomorfólogos, es un factor a tener muy en cuenta antes de presuponer la perdurabilidad de los espacios agrícolas y más cuando éstos, como ocurre en el Bajo Segura, fueron abandonados o transformados total-

\footnotetext{
19 Schiøler presenta un dibujo $(1973,97)$, mientras que una fotografía etnográfica de las piezas en uso en una noria puede verse en la obra Las razas humanas, dirigida por Bosch Gimpera y publicada en dos volúmenes en Barcelona en I928 (vol. II, p.l83), lám. II.

20 H. Kirchner y C. Navarro, "Objetivos, métodos y práctica de la arqueología hidráulica", 1994, (p. 171, nota. 27), donde las autoras plantean la conveniencia de un estudio minucioso de los espacios agrarios cronológicamente discriminados a partir del estudio arqueológico de la cultura material y del poblamiento.
} 
mente a partir del siglo Xl, tras la planificación de un agrosistema más extenso, basado en la canalización de las aguas derivadas de azudes fluviales. ${ }^{21}$ De hecho, ni tan siquiera este segundo espacio hidráulico, continuamente ampliado hasta la actualidad, muestra un diseño inmutable, como testimonia Mosén Bellot en el siglo XVII a propósito de la alquería oriholana de Redován, de la que dice que a finales del siglo XV “...debía ser harta mejor tierra y más huerta de lo que es hoy, porque la cequia de escorratel iba por mucho más arriba; pero con las avenidas de la rambla crece y se alza la tierra y decrece la huerta faltándole el agua" (BELLOT, 1959, II, 181). Las peculiares condiciones de sedimentación de estas zonas bajas no impiden totalmente el estudio arqueológico de los campos cultivados, pero sí introducen un factor azaroso e involuntario en la investigación científica que se aprecia en el caso de la noria de "Les Jovades" de Oliva, construida en relación con un espacio agrario cuya superficie se halla hoy a casi dos metros por debajo del nivel actual y encontrada fortuitamente durante la explotación de una cantera de extracción de tierra (BAZZANA et alii, 1987, 15 y ss.).

En mi opinión, la caracterización geomorfológica de las llanuras de inundación obliga a reconsiderar o al menos matizar uno de los principios fundamentales de la arqueología hidráulica teórica, el principio de estabilidad de los espacios regados, que "...permite que ahora podamos estudiarlos y sea posible reconstruir su diseño original". ${ }^{22}$ Aunque el trabajo de campo arqueológico siempre es necesario, no conviene olvidar que los sistemas de regadío en los llanos de inundación no están obligatoriamente sujetos a los mismos principios de rigidez y estabilidad que caracterizan otros sistemas hidráulicos, como por ejemplo las terrazas irrigadas en zonas de pendiente, y que por tanto en estos ambientes el primer trabajo de campo ha de ser el geomorfológico, so pena de equivocar la estrategia y obtener conclusiones arqueológicas totalmente anacrónicas. Esta singular concurrencia de condiciones ambientales, sin duda "lamentable" desde un punto de vista arqueológico, puede determinar que en ocasiones los únicos indicios de la existencia de un espacio hidráulico imaginado sean estos humildes arcaduces. Convendremos entonces que los conocimientos históricos que se obtienen a partir de su estudio son cualitativamente muy importantes: concedámosles pues una categoría cientifica superior a la del fetiche. ${ }^{23}$

\section{BIBLIOGRAFÍA}

ACIÉN ALMANSA, M. y MARTÍNEZ MADRID, R. (1989): "Cerámica islámica arcaica del sureste de alAndalus", Boletín de Arqueología Medieval. Madrid, 3, pp. I23- 135.

ACIÉN, M.; CASTILLO, F., FERNÁNDEZ, $M^{a}$ ।., MARTÍNEZ, R., PERAL, C.; VALLEJO, A., (e. p.): "Evolución de los tipos cerámicos en el S.E de alAndalus", vème Colloque International sur la céramique médiévale en Méditerranée Occidentale. (Rabat, 1991).

AZUAR RUIZ, R. y GUTIERREZ LLORET, S. (e. p.): "Formación y transformación de un espacio agrícola islámico en el sur del Pais Valenciano: el Bajo Segura (siglos IX-XIII)", Castrum 5: Archéologie des espaces agraires méditerranéens au Moyen Age. Archéologie de l'habitat fortifié. (Murcia, 1992).

BARCELÓ PERELLÓ, M., ( 1989 ): "El diseño de espacios irrigados en al-Andalus: un enunciado de principios generales", I Coloquio de Historia y Medio Físico; el agua en zonas áridas: arqueología e historia (Almería, 1989), XIII-L, Almería.

BARCELÓ PERELLÓ, M., (1992): "Quina arqueologia per al-Andalus?", Coloquio Hispano-Italiano de Arqueología Medieval (Granada, 1990), pp. 243-252.

BAZZANA, A. 1979: "Céramiques médiévales: les méthodes de la description analytique apliquées aux productions de l'Espagne orientale", Melanges de la Casa de Velázquez, Madrid, XV, pp. I35- 185.

\footnotetext{
21 Nos referimos a la huerta de Orihuela, vinculada a esta madina. Sobre su formación puede verse el artículo de AZUAR, R. y GUTIÉRREZ, S. (e.p.): "Formación y transformación de un espacio agrícola islámico en el sur del País Valenciano: el caso del Bajo Segura (siglos IX-XIII)", Castrum V.

22 KIRCHNER y NAVARRO, 1994, I61; BARCELÓ, 1989, XXVII y 1992, 246.

23 M. BARCELÓ, intervención 18 del coloquio a las ponencias de M. ACIÉN y A. GÓMEZ en el I Encuentro de Arqueología y Patrimonio de Salobreña, La cerámica altomedieval en el sur de al- Andalus, 1993, Granada, p. 203.
} 
BAZZANA, A. 1987: "Una noria árabe en la huerta de Oliva (Valencia)", II Congreso de Arqueología Medieval Española, (Madrid, 1987), II, Madrid, pp. 42I-432.

BAZZANA, A.; CLIMENT, S.; MONTMESSIN, Y. (1987): El yacimiento medieval de "Les Jovades", Oliva (Valencia), Ayuntamiento de Oliva, Oliva.

BELLOT, P., (1956): Anales de Orihuela (siglos XIV-XVI); J. TORRES FONTES (Ed.), 2 vols., Orihuela.

BOSCH,C. y CHINCHILLA, M., (1987): "Formas cerámicas auxiliares: anafes, arcaduces y otras", II Congreso de Arqueología Medieval Española (Madrid, 1987), II, Madrid, pp. 529-38.

CARO BAROJA, J., (1983): "Norias, azudas, aceñas" y "Sobre la historia de la noria de tiro", Tecnología Popular Española. Artes del tiempo y del espacio, 6, Madrid, pp. 239-409.

CARRASCO MARTÍN, M‥ J., (1987): "Avance del estudio de la cerámica hispano-musulmana procedente de los "Baños de la Reina Mora" (Sevilla)", II Congreso de Arqueología Medieval Española (Madrid, 1987), II, Madrid, pp. $49 \mid-500$

GLICK, Th. F., (1977): "Noria pots in Spain", Technology and Culture, Vol. 18, No 4, pp. 644-650.

GLICK, Th. F., (1988): Regadío y sociedad en la Valencia medieval, Valencia.

GLICK, Th. F., (1989): "Las técnicas hidráulicas antes y después de la conquista", En torno al 750 aniversario. Antecedentes y consecuencias de la Conquista de Valencia, I, Valencia, pp. 53-7|

GUTIÉRREZ LLORET, S. (e. p.): Tudmīr: de la antigüedad tardía al mundo islámico, Madrid-Alicante.

GUTIÉRREZ LLORET, S. (1988): Cerámica común paleoandalusí del sur de Alicante (siglos VII-X). Alicante.

GUTIÉRREZ LLORET, S. (1992): "Espacio y poblamiento pa-leoandalusí en el sur de Alicante: origen y distribución". II Congreso de Arqueología Medieval Española (Oviedo, 1989), Oviedo, pp. 34I-348.

\section{RESUMEN}

Se presenta un tipo de arcaduz conocido solamente, hasta el momento, en el bajo Segura (MurciaAlicante). Éste aparece por primera vez a mitad del S. VIII, y su tipología evoluciona durante los S. IX y X. Se trata del arcaduz emiral más antiguo documentado por la Arqueología. Su incorporación a los repertorios cerámicos de tradición indígena hacen de él un indicador del contacto con las nuevas poblaciones egipcias establecidas en la región.

PALABRAS CLAVE: Cerámica, Noria, regadío, Tudmir, emirato.
GUTIÉRREZ LLORET, S. (1993): "La cerámica paleoandalusí del sureste peninsular (Tudmîr): producción y distribución (siglos VII al X)", La cerámica altomedieval en el sur de al-Andalus (Salobreña, 1990), Granada, pp. 37-66,

GUTIÉRREZ LLORET, S., (1995): "El origen de la huerta de Orihuela entre los siglos VII y XI: una propuesta arqueológica sobre la explotación de las zonas húmedas del Bajo Segura", Arbor, mayo, № 593, Madrid, pp. 65-94.

KIRCHNER, H. y NAVARRO, C., (1994): "Objetivos, métodos y práctica de la arqueología hidráulica", Arqueología y territorio medieval, Jaén, I, I 59- 182.

LUZÓN, J. M., (1968): "Los sistemas de desagüe en las minas romanas del Suroeste peninsular", Archivo Español de Arqueología, Madrid, vol. 4I, No I I7-II8, pp. I0I-I20.

MATEU, J., (1989): "Assuts i vores fluvials regades al País Valencià medieval", Los paisajes del agua. Libro jubilar dedicado al profesor A. López Gómez, Universitat de València-Universitat d'Alacant, pp. |65-| 85.

MUÑOZ LÓPEZ, F., (1992): "Nuevos datos sobre urbanismo y alfarería medieval en Murcia", Verdolay, 4, pp. 175- 184 .

POZO MARTINEZ, I., (|99|): "Un baño privado islámico en la calle Polo Medina (Murcia)", Verdolay, 3, Murcia, pp. 79-94.

RETUERCE VELASCO, M., (1990): Cerámica islámica en la comunidad de Madrid", Madrid del siglo IX al XI, Madrid, pp. 145-164.

ROSSELLÓ BORDOY, G., (1978): Ensayo de sistematización de la cerámica árabe en Mallorca, Palma de Mallorca.

ROSSELLÓ BORDOY, G., (1991): El nombre de las cosas en al-Andalus: una propuesta de terminología cerámica, Palma de Mallorca.

ROSSELLÓ VERGER, V., (1989): "Los llanos de inundación". Avenidas fluviales e inundaciones en la cuenca del Mediterráneo, Alicante, pp. 243-283.

SCHIØLER, T., ( 1973): Roman and Islamic water-lifting Wheels, Lund.

\section{ABSTRACT}

On présente un type d'auge seulement connue, jusq'à présent dans le Bas Segura (Murcie-Alicante). Celle-ci apparaît pour la première fois vers la moitié du VIIlème siècle, et sa typologíe évolue durant le IXème et le $X^{\text {ème }}$ siècle. II s'agit de la plus ancienne auge émirale documentée par l'archéologie. Son incorporation aux répertoires céramiques à tradition indigène, font d'elle un indicateur du contact avec les nouvelles populations égypciennes établies dans la région.

PAROLES CLÉES: Céramique, roue hydraulique à auges, irrigation, Tudmir, émirat. 


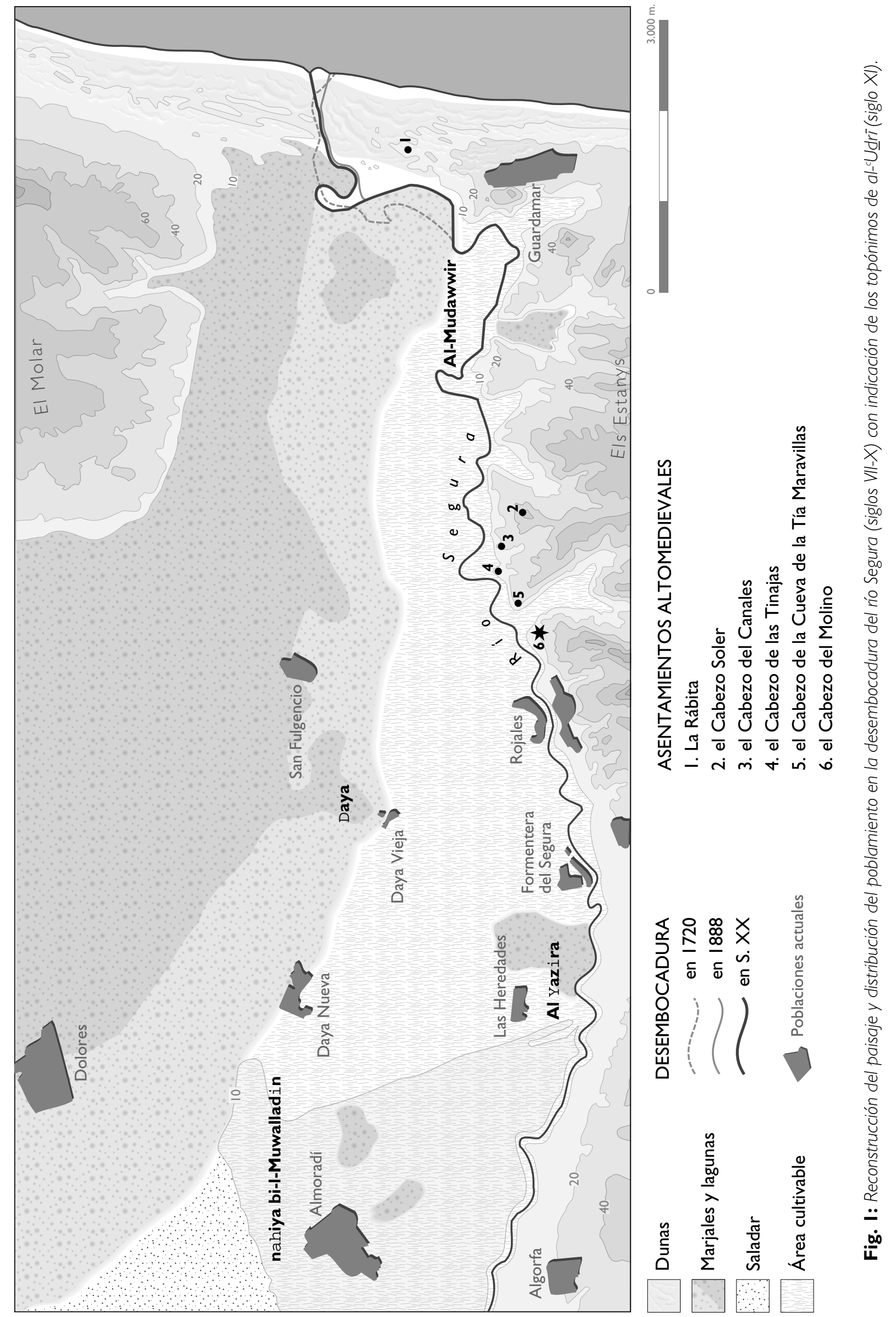




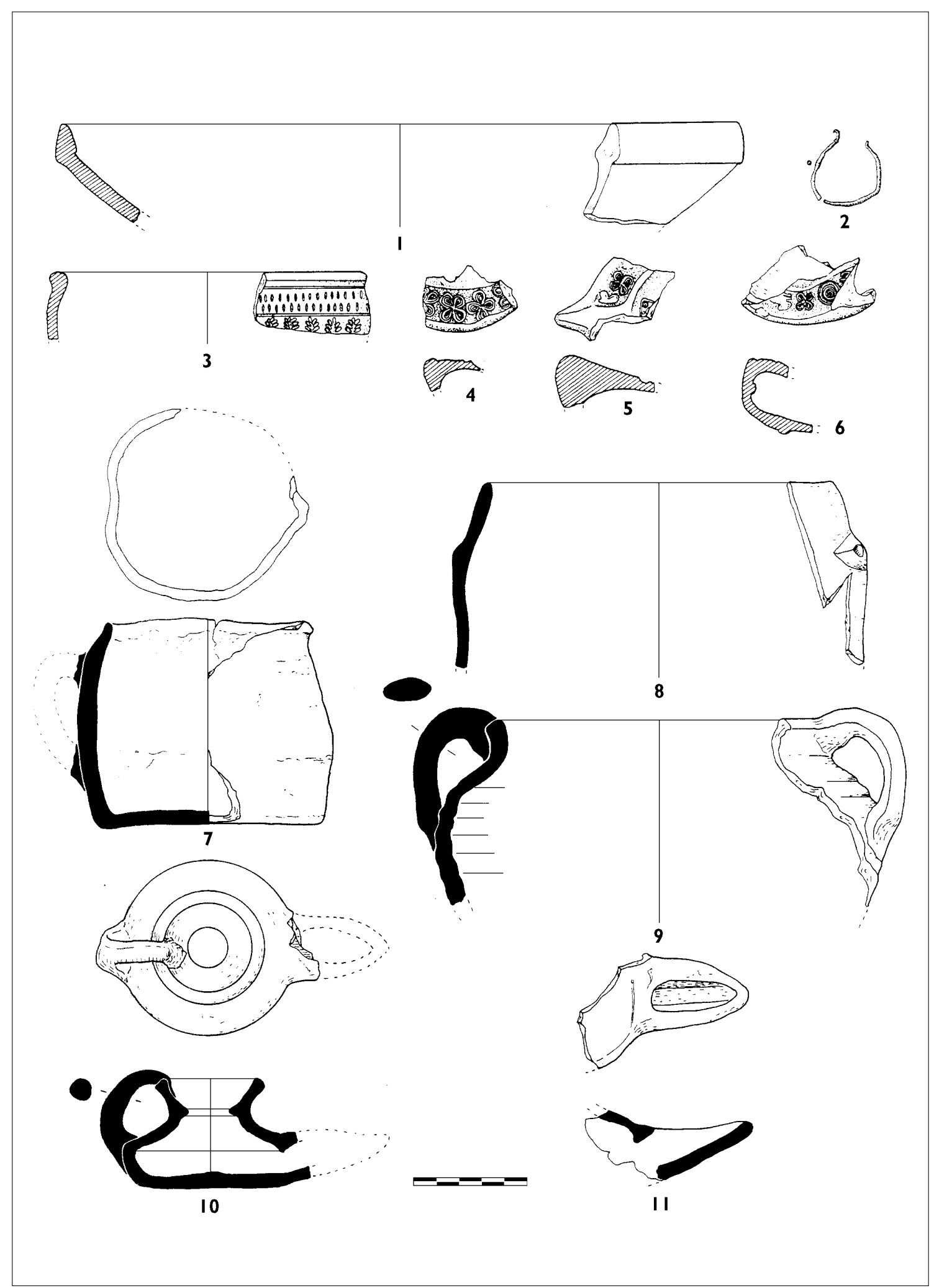

Fig. 2: Materiales del Cabezo del Molino (Rojales, Alicante).

I a 6: materiales tardorromanos (I y 4 a 6.- Terra sigillata africana; 2.- pendiente procedente del enterramiento; 3.- cerámica narbonense). 7 a I I: cerámicas emirales (7 y 8.- marmitas modeladas a mano; 9.- marmita a torno; 10 y 11 .- candiles) 


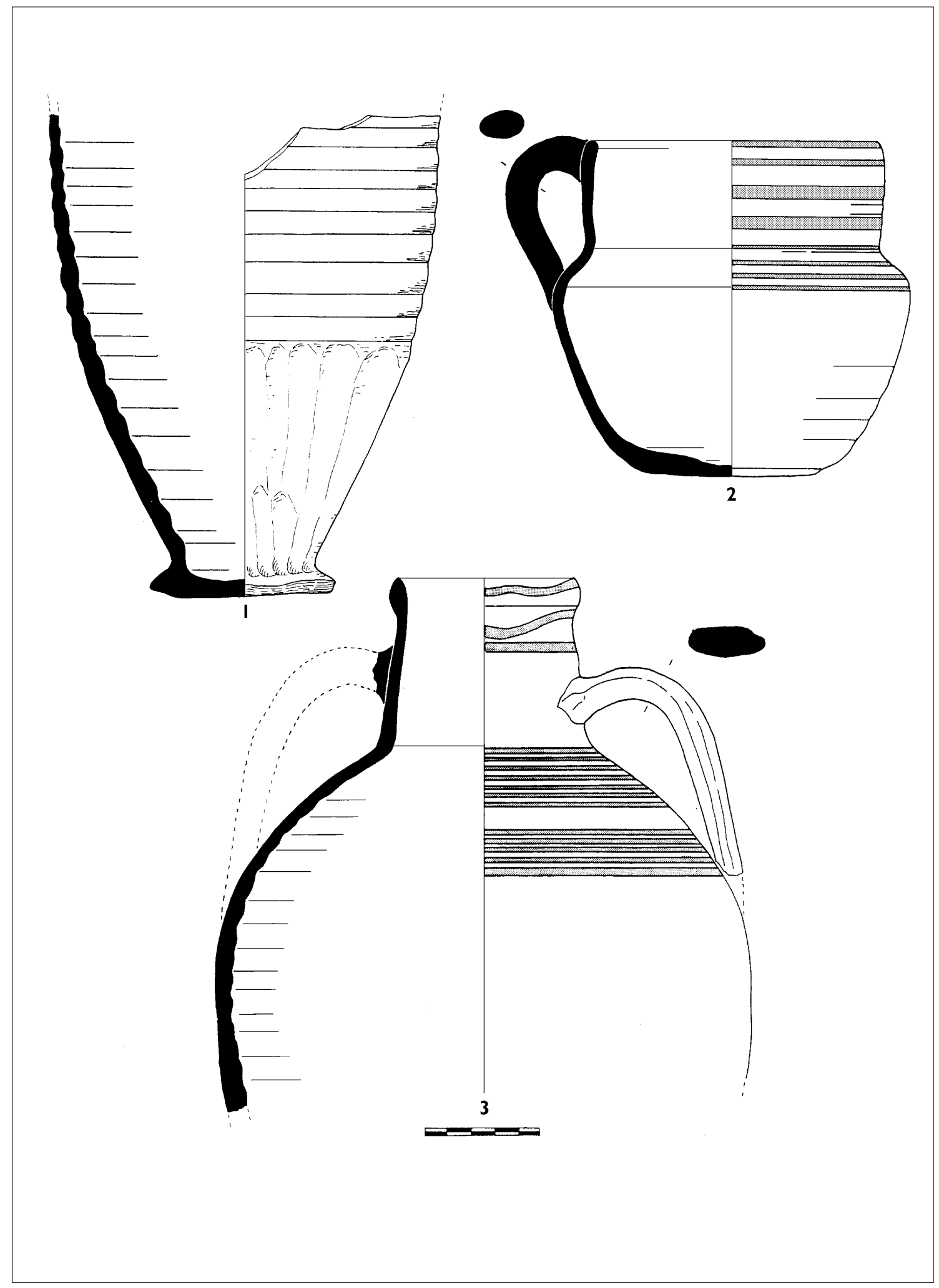

Fig. 3: Materiales del Cabezo del Molino (Rojales, Alicante).

I a 3: cerámicas emirales ( I.- arcaduz; 2 y 3.- jarrito y jarra pintados en óxido de hierro). 

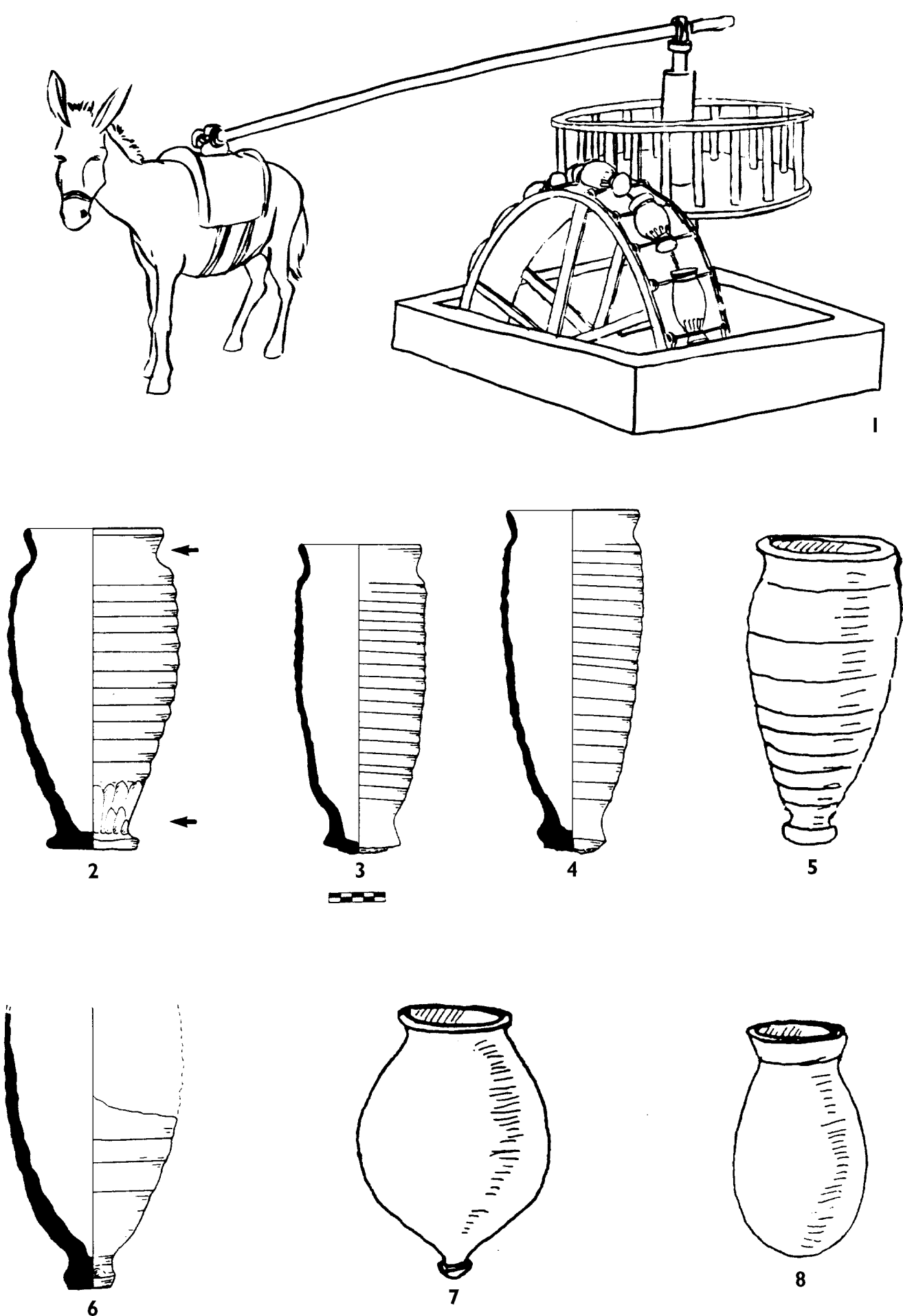

Fig. 4:

I: Representación de una cenia de tracción animal con el tipo de arcaduces documentados en este estudio (según LÓPEZ PADILLA). 2: reconstrucción de un arcaduz del Cabezo del Molino en Rojales, Alicante, con los puntos de fijación superior e inferior señalados y los característicos recortes en la parte baja del recipiente. 3 y 4: arcaduces de la Cl Cortés, Murcia (según MUÑOZ LOPEZ, 1993, figs. 3.5 y 3.6). 6: arcaduz de la Rábita de Guardamar del Segura (Alicante). 5, 7 y 8: arcaduces egipcios actuales (según SCHIØLER, 1973, 97) 


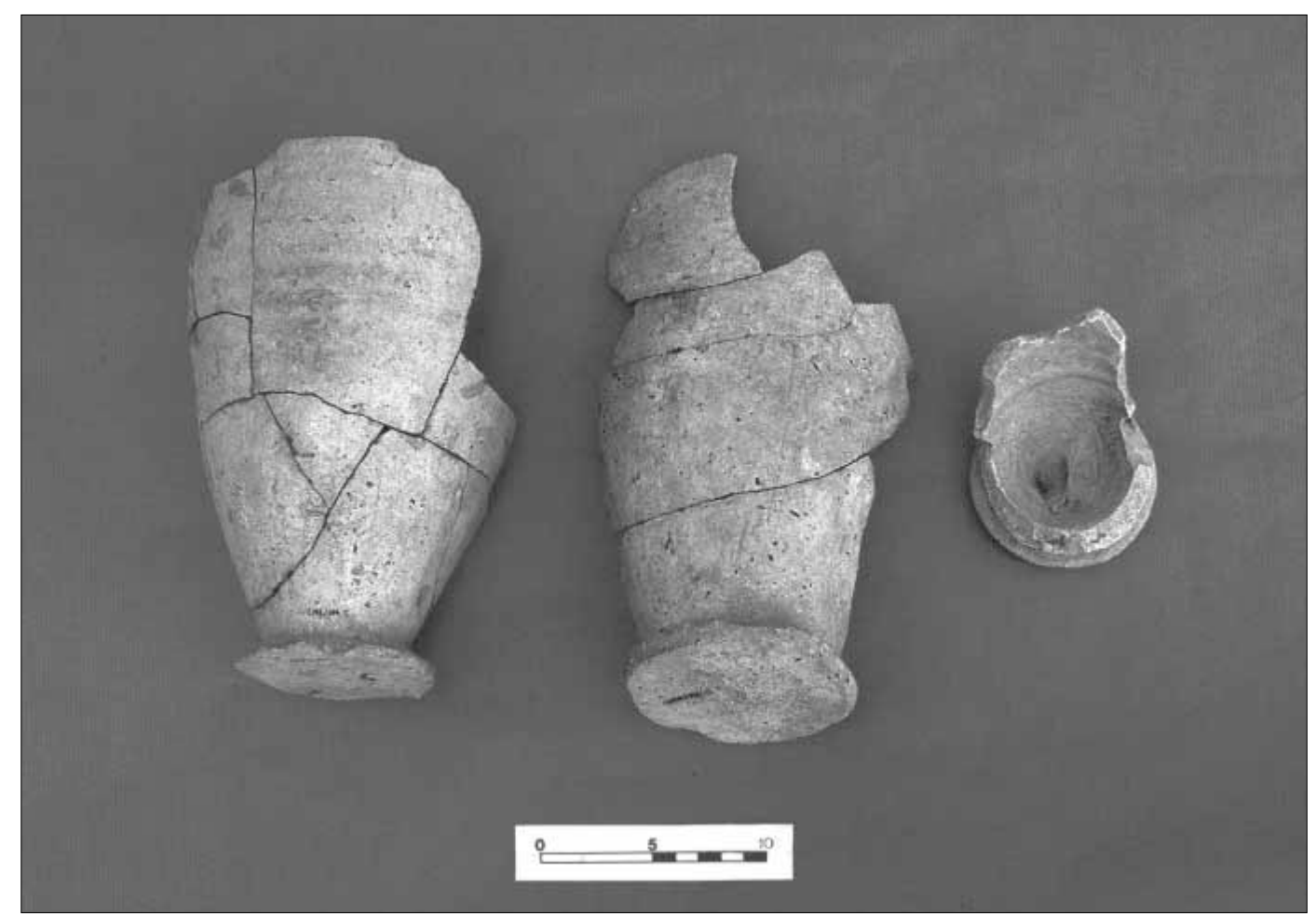

Lám. I: Arcaduces del Cabezo del Molino (Rojales, Alicante)

Lám. II: Noria a orillas del río Nilo (Egipto), Fot. BOYER; fuente: Las razas humanas, dirigida por P. BOSCH GIMPERA, Vol. Il, p. 193, Publicaciones del Instituto Gallach de Librería y Ediciones, Barcelona, 2 vols., 1928. Obsérvense las semejanzas tipológicas entre los arcaduces que forman la cadena de la noria egipcia y los ejemplares del Bajo Segura.

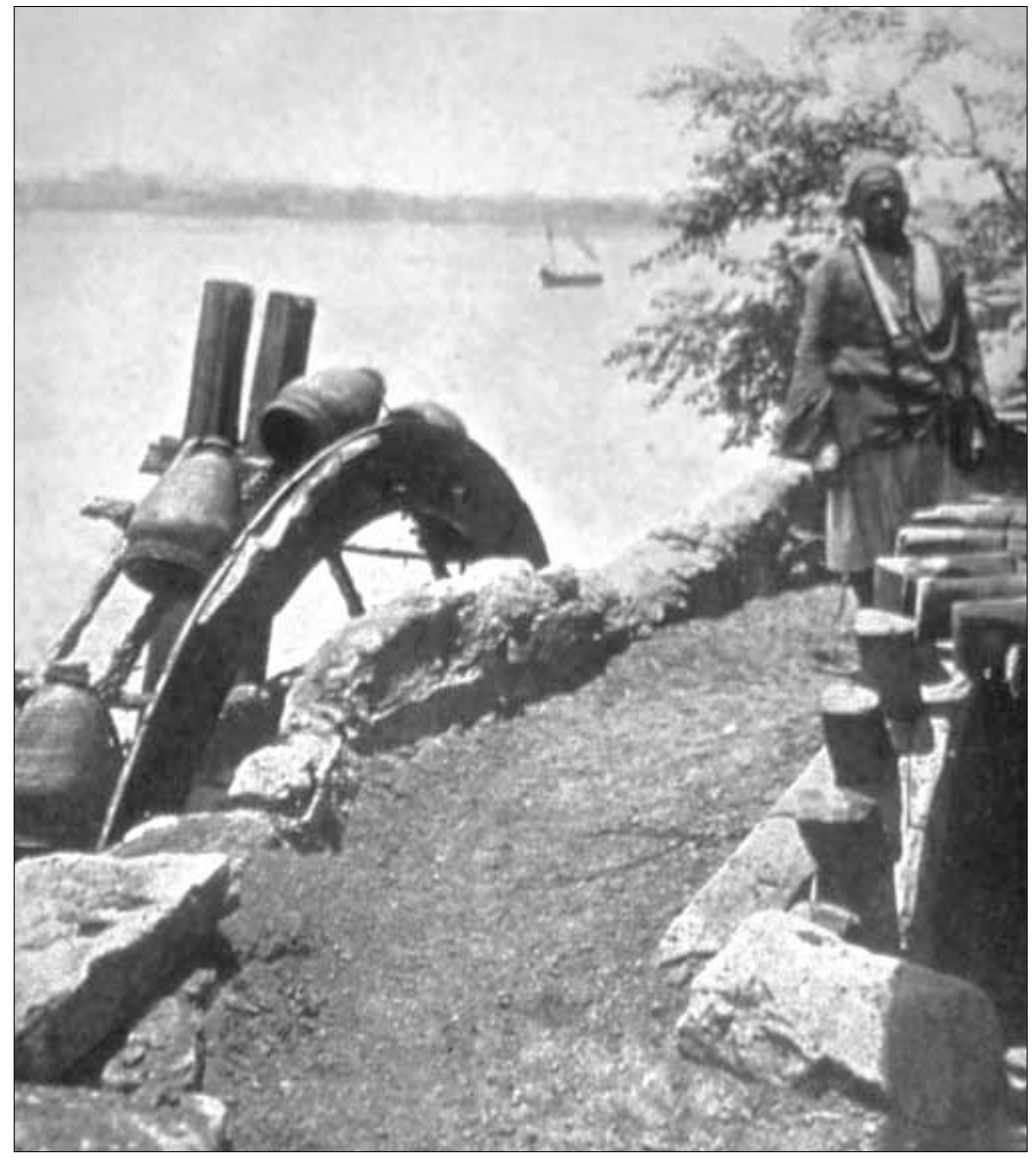

